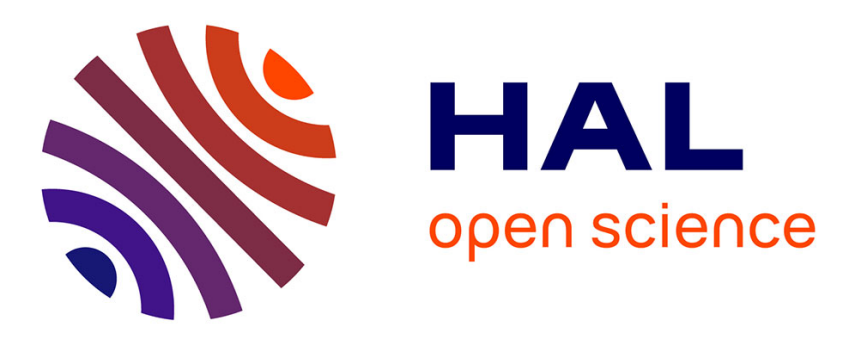

\title{
Passive harmonic mode locking of soliton crystals
}

Foued Amrani, Alioune Niang, Mohamed Salhi, Andrey Komarov, Hervé Leblond, François Sanchez

\section{To cite this version:}

Foued Amrani, Alioune Niang, Mohamed Salhi, Andrey Komarov, Hervé Leblond, et al.. Passive harmonic mode locking of soliton crystals. Optics Letters, 2011, 36 (21), pp.4239-4241. 10.1364/OL.36.004239 . hal-03187585

\section{HAL Id: hal-03187585 \\ https://univ-angers.hal.science/hal-03187585}

Submitted on 1 Apr 2021

HAL is a multi-disciplinary open access archive for the deposit and dissemination of scientific research documents, whether they are published or not. The documents may come from teaching and research institutions in France or abroad, or from public or private research centers.
L'archive ouverte pluridisciplinaire HAL, est destinée au dépôt et à la diffusion de documents scientifiques de niveau recherche, publiés ou non, émanant des établissements d'enseignement et de recherche français ou étrangers, des laboratoires publics ou privés. 


\title{
Passive harmonic mode locking of soliton crystals
}

\author{
Foued Amrani,* Alioune Niang, Mohamed Salhi, Andrey Komarov, \\ Herve Leblond, and François Sanchez \\ Laboratoire de Photonique d'Angers EA 4644, Université d'Angers, 2 Bd Lavoisier, 49000 Angers, France \\ ${ }^{*}$ Corresponding author: foued.amrani@gmail.com
}

Received June 27, 2011; revised September 13, 2011; accepted September 21, 2011;

posted September 26, 2011 (Doc. ID 149869); published October 27, 2011

\begin{abstract}
We report experimental observation of passive harmonic mode locking (HML) in which the basic pattern is a soliton crystal. Several crystal states were generated from an initial large bound state by increasing the pump power. The soliton crystals are identical and progressively span along the cavity to finally take a regular spacing leading to HML of solitons crystal. (C) 2011 Optical Society of America

OCIS codes: $140.3510,140.7090,060.5530$.
\end{abstract}

In recent years, passively mode-locked fiber lasers have demonstrated their ability to generate a large number of coexisting pulses up to several hundreds, especially in the anomalous dispersion [1]. Various passive modelocking techniques are used, such as the nonlinear loop mirror-based method [2], and the nonlinear polarization rotation (NLPR) technique [3]. The number of solitons is related to the high pumping power available in fiber lasers combined with the quantization of the soliton energy in the anomalous dispersion regime [4]. The evolution of the number of pulses versus the pumping power was experimentally observed by Richardson et al. [5]. Tang et al. studied the growth of the number of pulses one by one at various levels of pumping power and the related hysteresis phenomenon when the pumping power is decreased. They demonstrated by numerical simulations that multiple soliton formation in the laser is caused by a peak power-limiting effect of the laser cavity [6]. So the soliton energy quantization of the laser is a natural consequence of the gain competition between the solitons in the cavity. The hysteresis phenomenon was also reported by Komarov et al. [7]. They studied theoretically the multiple soliton operation and pump hysteresis of the soliton fiber lasers mode locked using the NLPR technique. Grelu and Soto-Crespo simulated numerically the multiple-pulse operation of the soliton fiber lasers []. Based on a propagation model, they reproduced the multipulse states observed experimentally. The multisoliton formation is only obtained for some specific sets of parameters. In the last few years, the increase in pumping power has enabled one to generate more and more coexisting solitons. Under such circumstances, solitons interact together, leading to the formation of temporal patterns (or longitudinal spatial patterns if we look at the distribution of the solitons along the cavity). These structures can exhibit some order or can be completely disordered, depending on the nature and the range of the interaction $[2,3,9]$. Several physical effects have been discussed in the literature and proposed to explain the various pulse ordering behaviors. Bound states and harmonic mode locking (HML) have been the matter of intensive research. A bound state of solitons consists in several identical pulses close together in one package, which are equidistant and have a fixed phase relation. The study of such states was initiated in 1991 by Malomed [10]. Akhmediev et al. found theoretically stable soliton pairs and trains within the Ginzburg-Landau equation
[11]. Tang et al. reported in 2001 the experimental observation of a stable bound state of two solitons separated by $1.16 \mathrm{ps}$ [12]. Grelu et al. obtained bound states of solitons with separations between solitons ranging from 2.7 to $10 \mathrm{ps}$ [13]. The scaling up of the power in fiber lasers has recently permitted the experimental observation of a bound state of hundreds of solitons, a stable crystal of 350 solitons [1]. On the other hand, among the different hypotheses proposed to explain the formation of the HML $[14,15]$, it seems that the interaction of pulses through a cw component is responsible for high-order HML [16,17]. In addition, it has been recently demonstrated that a small cw component in the spectrum enables one to control the sign and the amplitude of the soliton interaction [18]. Tang et al. reported the experimental observation of passive HML of soliton bunches and twin-pulse solitons in a passively mode-locked fiber ring laser $[19,20]$. Kutz et al. studied the effects of the gain depletion and recovery on the temporal spacing of the soliton pulses. They have shown that gain depletion in conjunction with its recovery provides an effective repulsive force between adjacent solitons by imparting a group velocity drift proportional to the interpulse spacing [14]. In this Letter, we report a remarkable soliton pattern formation. It consists in the harmonic distribution of soliton crystals. The evolution of the dynamics versus the pumping power is analyzed.

The experimental setup is schematically represented in Fig. 1. It is an all-fiber unidirectional ring cavity. Mode locking is achieved through NLPR technique. We use a double-clad Er:Yb $10 \mathrm{~W}$ fiber amplifier operating at $\lambda=1.55 \mu \mathrm{m}$ manufactured by Keopsys. It consists of a $5 \mathrm{~m}$ long double-clad fiber (DCF) that has a chromatic

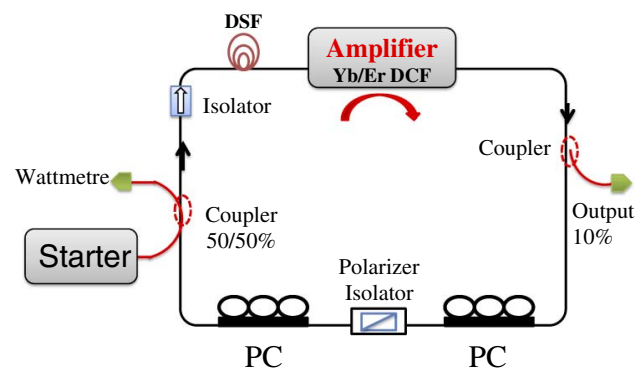

Fig. 1. (Color online) Experimental setup. DCF, double-clad fiber; DSF, dispersion-shifted fiber; PC, polarizer controller; starter, auxiliary laser to start up of the amplifier. 
dispersion coefficient $\beta_{2}^{\mathrm{DCF}}=-0.021 \mathrm{ps}^{2} / \mathrm{m}$ pumped at $980 \mathrm{~nm}$ by laser diodes emitting about $40 \mathrm{~W}$. The fibers DCF and SMF28 operate in the anomalous dispersion regime. A piece of dispersion-shifted fiber $\left(\beta_{2}^{\mathrm{DSF}}=\right.$ $0.14 \mathrm{ps}^{2} / \mathrm{m}$ ) is added to control the total cavity dispersion. To favor multiple-pulse mode locking, the total dispersion is set in the anomalous regime with $\beta_{2}^{\mathrm{TOT}}=$ $-0.12 \mathrm{ps}^{2}$, with a total cavity length of $30.5 \mathrm{~m}$ corresponding to a round trip time of $152.9 \mathrm{~ns}$. Because of internal electronic security to avoid irreversible damages of the $10 \mathrm{~W}$ amplifier in the absence of input signal, an auxiliary laser must be used to start up the amplifier. The external light is launched with a 50/50 coupler to the principal cavity with a signal power of $17 \mathrm{dBm}$. After the principal laser is operating, we turn off the starting laser to avoid any slave-master coupling between the two lasers. A polarizing isolator is set between two polarization controllers. Mode locking is obtained through the adjustment of the polarization controllers. The output intensity is detected with a high-speed photodetector (TIA-1200) and visualized with a fast oscilloscope (Tektronix TDS $6124 \mathrm{C}, 12 \mathrm{GHz}, 40 \mathrm{GSa}$ ). The spectral properties are analyzed with an optical spectrum analyzer (Anritsu MS 9710C) and the pulse duration is measured with an optical autocorrelator with a scanning range of $\pm 100 \mathrm{ps}$ (Femtochrome FR-103 XL).

At $15 \mathrm{~W}$ pumping level ( $4 \mathrm{~W}$ intracavity at $1.55 \mu \mathrm{m}$ ) and adjusting the polarization controllers, a condensate state is formed: it consists in a large number of regularly spaced identical solitons with constant relative phase differences from one round trip to the next. It is a soliton crystal of several hundreds of solitons [1]. The soliton train spans over $9.3 \mathrm{~ns}$, as indicated by the temporal intensity recorded in Fig. 2. The corresponding optical spectrum given in Fig. 3 proves the strong coherence between pulses since it is modulated with a contrast exceeding $95 \%$. The spectral period is $0.83 \mathrm{~nm}$, which corresponds to a temporal separation of $\sim 10$ ps between solitons, in good agreement with the autocorrelation trace shown in Fig. 4. From the train duration and the delay between solitons, we estimate the number of pulses in the soliton crystal to be 930 pulses, which is actually a record in comparison to our previous works [1-3]. The increase of the number of pulses in the crystal results

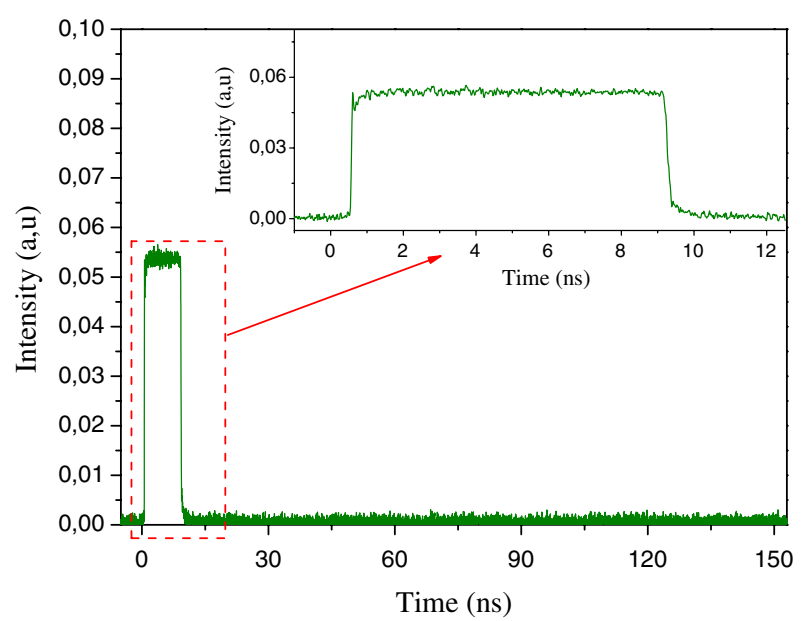

Fig. 2. (Color online) Temporal trace of a soliton crystal.

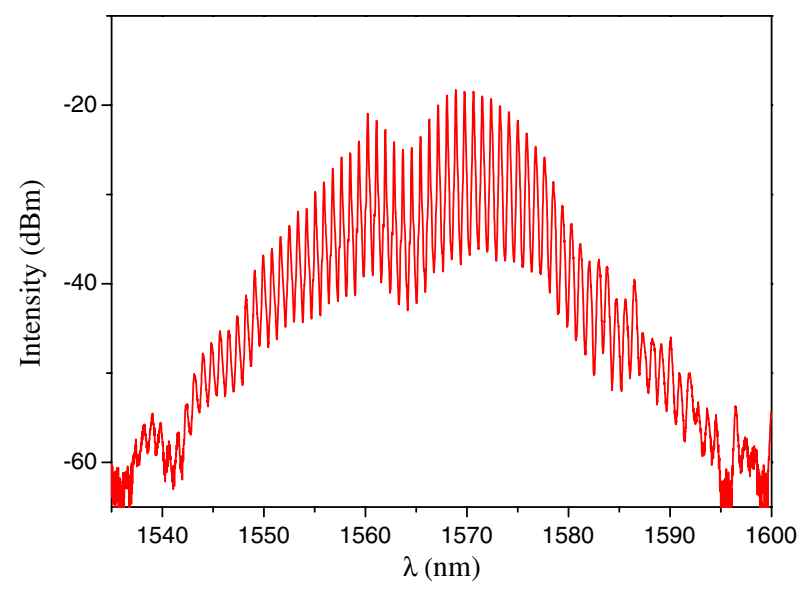

Fig. 3. (Color online) Optical spectrum of a soliton crystal.

from the growth of the pumping power as it was theoretically predicted [21].

By increasing slowly the pump power up to $25 \mathrm{~W}$ ( $6.3 \mathrm{~W}$ intracavity at $1.55 \mu \mathrm{m}$ ), several soliton packets begin to become loose from the initial soliton crystal. These packets of pulses tend to become loose from the initial train of pulses. Just when several soliton packets begin to loosen from the initial soliton crystal, a cw component appears in the optical spectrum. The CW component introduces a repulsive force between solitons. Gradually, the soliton packets spread over a large part of the cavity and finally fill the whole cavity, as displayed in Fig. 5. The inset in Fig. 5 reveals that the packets are uniformly distributed and that all packets have the same time duration. Each packet is a bound state as demonstrated by the strongly modulated optical spectrum of Fig. $\underline{6}$. The spectral period is $0.82 \mathrm{~nm}$, resulting in a delay between solitons inside the bound state of $10 \mathrm{ps}$, as confirmed by the autocorrelation trace shown in Fig. 7 . We note that the envelope of the autocorrelation trace is nearly triangular, which proves that the solitons are identical inside a packet. From the delay and the duration of a bound state, we estimate the number of solitons inside a bound state to be about 50. The bound state is therefore a soliton crystal containing only a small part of the total soliton set. We obtain finally the fiftieth harmonic of soliton crystals containing about 50 solitons. The total number of solitons is

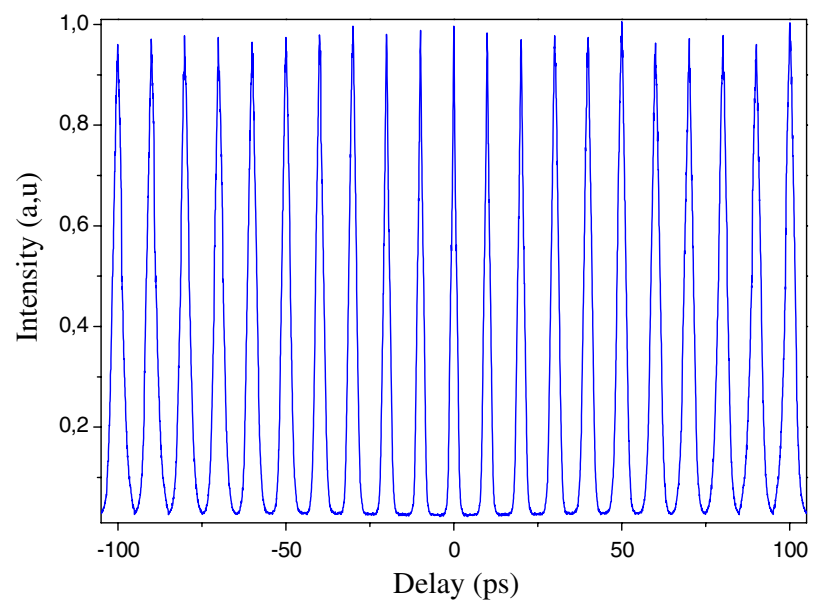

Fig. 4. (Color online) Autocorrelation trace of a soliton crystal. 


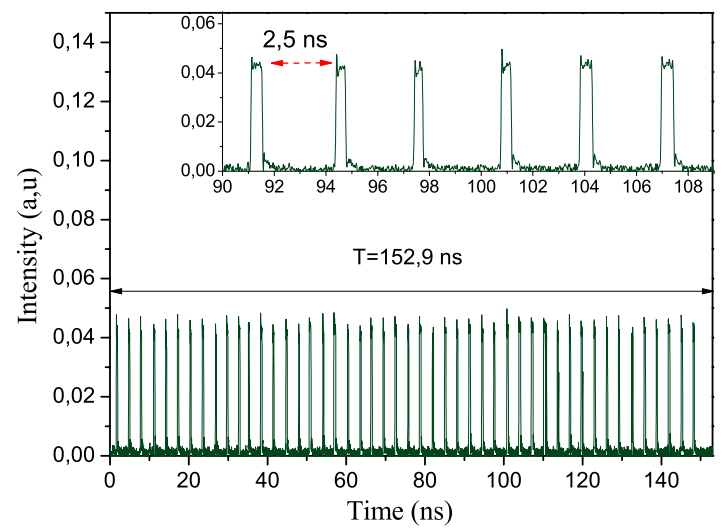

Fig. 5. (Color online) Temporal trace of HML of solitons crystal.

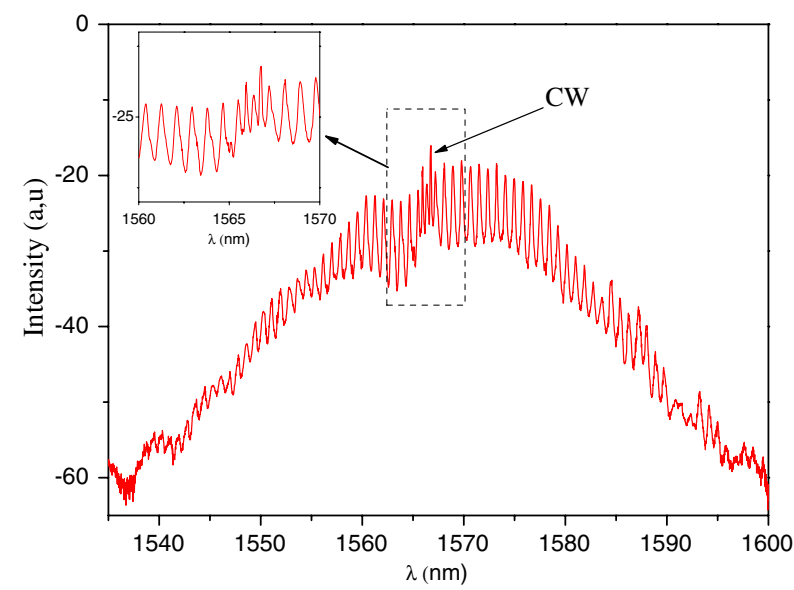

Fig. 6. (Color online) Optical spectrum of HML of solitons crystal.

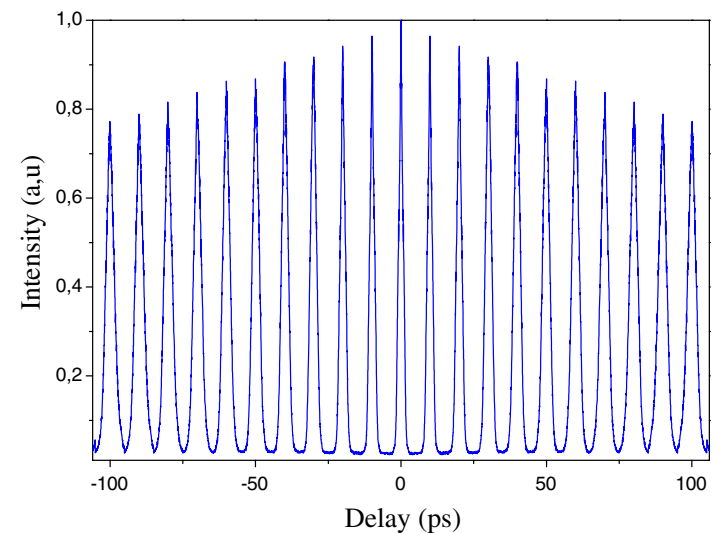

Fig. 7. (Color online) Autocorrelation trace of HML of solitons crystal.

therefore about 2500. Inspecting of the optical spectrum reveals two remarks. First, the modulation characterizes the bound state, and, second, the HML is correlated with the existence of a cw component visible in the optical spectrum as it was previously indicated [16]. The HML state is stable over $1 \mathrm{~h}$ and is reproducible. The radiofrequency spectrum calculated by the Fourier transform of the temporal trace reveals that the repetition rate is about $330 \mathrm{MHz}$ and that supermode suppression is better than $15 \mathrm{~dB}$. Compared with the fundamental cavity frequency $6.53 \mathrm{MHz}$, it proves the generation of the fiftieth harmonic. The timing jitter between crystals is deduced from the histogram of the corresponding delays; it is about $200 \mathrm{ps}$. Their amplitude fluctuation, about $10 \%$, is deduced from the temporal trace. The pulse to pulse timing jitter inside a crystal can be estimated from the broadening of the peaks of the autocorrelation trace from the center to the edge. It is less than $0.4 \mathrm{ps}$.

In summary we have experimentally demonstrated the HML of soliton crystals in an Er-doped DCF laser mode locked by NLPR. The formation dynamics is obtained by increasing the pumping power, the initial soliton crystal splits into several identical soliton crystals of smaller extent. The splinters rearrange their relative positions in the cavity and then form the harmonically mode-locked state. The existence of a cw component has been pointed out and is in agreement with previous theoretical predictions. The initial goal of this work was to scale up the pumping power in order to considerably increase the number of pulses in the crystal state.

We are grateful to D. Mihalache for his comments on the manuscript. We thank the Agence Nationale de la Recherche for supporting this work (contract ANR-2010BLANC-0417-01-SOLICRISTAL).

\section{References}

1. A. Haboucha, H. Leblond, M. Salhi, A. Komarov, and F. Sanchez, Opt. Lett. 33, 524 (2008).

2. F. Amrani, M. Salhi, P. Grelu, H. Leblond, and F. Sanchez, Opt. Lett. 36, 1545 (2011).

3. F. Amrani, A. Haboucha, M. Salhi, H. Leblond, A. Komarov, and F. Sanchez, Appl. Phys. B 99, 107 (2010).

4. A. B. Grudinin, D. J. Richardson, and D. N. Payne, Electron. Lett. 28, 67 (1992).

5. D. J. Richardson, R. I. Laming, D. N. Payne, V. J. Matsas, and M. W. Phillips, Electron. Lett. 27, 1451 (1991).

6. D. Y. Tang, L. M. Zhao, B. Zhao, and A. Q. Liu, Phys. Rev. A 72, 043816 (2005).

7. A. Komarov, H. Leblond, and F. Sanchez, Phys. Rev. A 71, 053809 (2005).

8. P. Grelu and J. M. Soto-Crespo, J. Opt. B 6, S271 (2004).

9. F. Amrani, M. Salhi, H. Leblond, A. Haboucha, and F. Sanchez, Opt. Express 19, 13134 (2011).

10. B. A. Malomed, Phys. Rev. A 44, 6954 (1991).

11. N. N. Akhmediev, A. Ankiewizc, and J. M. Soto-Crespo, Phys. Rev. Lett. 79, 4047 (1997).

12. Y. D. Gong, P. Shum, T. Hiang, C. Q. Wen, and D. Y. Tang, Opt. Commun. 200, 389 (2001).

13. P. Grelu, F. Belhache, F. Gutty, and J. M. Soto-Crespo, Opt. Lett. 27, 966 (2002).

14. J. N. Kutz, B. C. Collings, K. Bergman, and W. H. Knox, IEEE J. Quantum Electron. 34, 1749 (1998).

15. S. Zhou, D. G. Ouzounov, and F. Wise, Opt. Lett. 31, 1041 (2006).

16. F. Amrani, A. Haboucha, M. Salhi, H. Leblond, A. Komarov, P. Grelu, and F. Sanchez, Opt. Lett. 34, 2120 (2009).

17. Z. X. Zhang, L. Zhan, X. X. Yang, S. Y. Luo, and Y. X. Xia, Laser Phys. Lett. 4, 592 (2007).

18. A. Komarov, K. Komarov, H. Leblond, and F. Sanchez, J. Opt. A 9, 1149 (2007).

19. B. Zhao, D. Y. Tang, P. Shum, W. S. Man, H. Y. Tam, Y. D. Gong, and C. Lu, Opt. Commun. 229, 363 (2004).

20. L. M. Zhao, D. Y. Tang, and D. Liu, Appl. Phys. B 99, 441 (2010).

21. A. Komarov, A. Haboucha, and F. Sanchez, Opt. Lett. 33, 2254 (2008). 